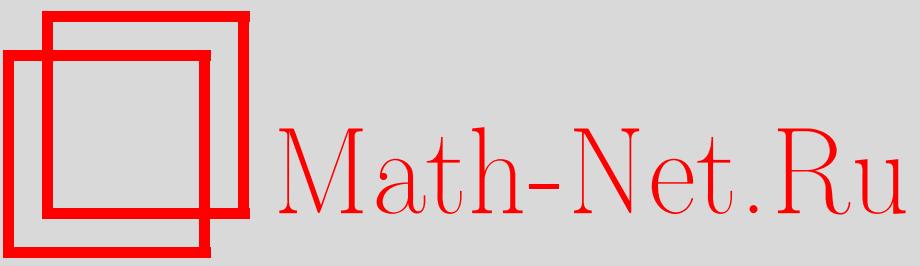

В. П. Маслов, Надвторичное квантование и квантование энтропии при сохранении заряда, УМH, 2000, том 55, выпуск 6, 145-146

DOI: https://doi.org/10.4213/rm347

Использование Общероссийского математического портала Math-Net.Ru подразумевает, что вы прочитали и согласны с пользовательским соглашением

http://www . mathnet.ru/rus/agreement

Параметры загрузки:

IP : 3.93.64.190

26 апреля 2023 г., 04:43:12 


\title{
НАДВТОРИЧНОЕ КВАНТОВАНИЕ И КВАНТОВАНИЕ ЭНТРОПИИ ПРИ СОХРАНЕНИИ ЗАРЯДА
}

\author{
В. П. МАСЛОВ
}

Пусть $n_{1}, n_{2}, m \geqslant 0, j_{k}, i_{s}$ - целые числа. Рассмотрим функции

$$
\Phi_{n, m_{1}, m_{2}}\left(x_{1}, j_{1}, \ldots, x_{n_{1}}, j_{n_{1}}, y_{1}, i_{1}, \ldots, y_{n_{2}}, i_{n_{2}}, z_{1}, w_{1}, \ldots, z_{m}, w_{m}\right),
$$

симметричные относительно перестановок пар переменных $x_{p}, j_{p}$ и $x_{q}, j_{q} ; y_{r}, i_{r}$ и $y_{s}, i_{s} ; z_{l}, w_{l}$ и $z_{t}, w_{t}$.

Пусть $\mathscr{F}$ - соответствующее пространство Фока [1], [2] и в нем вводятся операторы рождения и уничтожения $\widehat{b}_{1}^{ \pm}(x, j), \widehat{b}_{2}^{ \pm}(y, i), \widehat{B}^{ \pm}(z, w)$ :

$$
\begin{aligned}
& \left(\widehat{b}_{1}^{+}(x, j) \Phi\right)_{n_{1}, n_{2}, m}\left(x_{1}, j_{1}, \ldots, x_{n_{1}}, j_{n_{1}}, y_{1}, i_{1}, \ldots, y_{n_{2}}, i_{n_{2}}, z_{1}, w_{1}, \ldots, z_{m}, w_{m}\right) \\
& =\frac{1}{\sqrt{n_{1}}} \sum_{l=1}^{n_{1}} \delta_{j j_{l}} \delta\left(x-x_{l}\right) \Phi_{n_{1}-1, n_{2}, m}\left(x_{1}, j_{1}, \ldots, x_{l-1}, j_{l-1}, x_{l+1}, j_{l+1}, \ldots, x_{n_{1}}, j_{n_{1}},\right. \\
& \left.y_{1}, i_{1}, \ldots, y_{n_{2}}, i_{n_{2}}, z_{1}, w_{1}, \ldots, z_{m}, w_{m}\right) \text {, } \\
& \left(\widehat{b}_{1}^{-}(x, j) \Phi\right)_{n_{1}, n_{2}, m}\left(x_{1}, j_{1}, \ldots, x_{n_{1}}, j_{n_{1}}, y_{1}, i_{1}, \ldots, y_{n_{2}}, i_{n_{2}}, z_{1}, w_{1}, \ldots, z_{m}, w_{m}\right) \\
& =\sqrt{n_{1}+1} \Phi_{n_{1}+1, n_{2}, m}\left(x, j, x_{1}, j_{1}, \ldots, x_{n_{1}}, j_{n_{1}}, y_{1}, i_{1}, \ldots, y_{n_{2}}, i_{n_{2}}, z_{1}, w_{1}, \ldots, z_{m}, w_{m}\right), \\
& \left(\widehat{b}_{2}^{+}(y, i) \Phi\right)_{n_{1}, n_{2}, m}\left(x_{1}, j_{1}, \ldots, x_{n_{1}}, j_{n_{1}}, y_{1}, i_{1}, \ldots, y_{n_{2}}, i_{n_{2}}, z_{1}, w_{1}, \ldots, z_{m}, w_{m}\right) \\
& =\frac{1}{\sqrt{n_{2}}} \sum_{l=1}^{n_{2}} \delta_{i i_{l}} \delta\left(y-y_{l}\right) \Phi_{n_{1}, n_{2}-1, m}\left(x_{1}, j_{1}, \ldots, x_{n_{1}}, j_{n_{1}}, y_{1}, i_{1}, \ldots, y_{l-1}, i_{l-1},\right. \\
& \left.y_{l+1}, i_{l+1}, \ldots, y_{n_{2}}, i_{n_{2}}, z_{1}, w_{1}, \ldots, z_{m}, w_{m}\right), \\
& \left(\widehat{b}_{2}^{-}(y, i) \Phi\right)_{n_{1}, n_{2}, m}\left(x_{1}, j_{1}, \ldots, x_{n_{1}}, j_{n_{1}}, y_{1}, i_{1}, \ldots, y_{n_{2}}, i_{n_{2}}, z_{1}, w_{1}, \ldots, z_{m}, w_{m}\right) \\
& =\sqrt{n_{2}+1} \Phi_{n_{1}, n_{2}+1, m}\left(j_{1}, \ldots, x_{n_{1}}, j_{n_{1}}, y, i, y_{1}, i_{1}, \ldots, y_{n_{2}}, i_{n_{2}}, z_{1}, w_{1}, \ldots, z_{m}, w_{m}\right) \text {, } \\
& \left(\widehat{B}^{+}(z, w) \Phi\right)_{n_{1}, n_{2}, m}\left(x_{1}, j_{1}, \ldots, x_{n_{1}}, j_{n_{1}}, y_{1}, i_{1}, \ldots, y_{n_{2}}, i_{n_{2}}, z_{1}, w_{1}, \ldots, z_{m}, w_{m}\right) \\
& =\frac{1}{\sqrt{m}} \sum_{l=1}^{m} \delta\left(z-z_{l}\right) \delta\left(w-w_{l}\right) \Phi_{n_{1}, n_{2}, m-1}\left(x_{1}, j_{1}, \ldots, x_{n_{1}}, j_{n_{1}}, y_{1}, i_{1}, \ldots, y_{n_{2}}, i_{n_{2}},\right. \\
& \left.z_{1}, w_{1}, \ldots, z_{l-1}, w_{l-1}, z_{l+1}, w_{l+1}, \ldots, z_{m}, w_{m}\right) \text {, } \\
& \left(\widehat{B}^{-}(z, w) \Phi\right)_{n_{1}, n_{2}, m}\left(x_{1}, j_{1}, \ldots, x_{n_{1}}, j_{n_{1}}, y_{1}, i_{1}, \ldots, y_{n_{2}}, i_{n_{2}}, z_{1}, w_{1}, \ldots, z_{m}, w_{m}\right) \\
& =\sqrt{m+1} \Phi_{n_{1}, n_{2}, m+1}\left(x_{1}, j_{1}, \ldots, x_{n_{1}}, j_{n_{1}}, y_{1}, i_{1}, \ldots, y_{n_{2}}, i_{n_{2}}, z, w, z_{1}, w_{1}, \ldots, z_{m}, w_{m}\right) \text {. }
\end{aligned}
$$

Рассмотрим теперь "обычное" фоковское пространство [3] $\mathscr{H}$ системы бозонов с зарядами \pm 1 , которое состоит из наборов функций $\phi=\left\{\phi_{q_{1}, q_{2}}\left(x_{1}, \ldots, x_{q_{1}}, y_{1}, \ldots, y_{q_{2}}\right)\right\}, q_{1}, q_{2}=0,1, \ldots$, симметричных относительно перестановок переменных $x_{l}$ и $x_{p}$ и относительно перестановок переменных $y_{k}$ и $y_{r}$. В этом пространстве определены операторы рождения и уничтожения бозонов с зарядом $1 \widehat{\psi}_{1}^{ \pm}(x)$ и бозонов с зарядом $-1 \widehat{\psi}_{1}^{ \pm}(y)$ :

$$
\begin{aligned}
& \left(\widehat{\psi}_{1}^{+}(x) \phi\right)_{q_{1}, q_{2}}\left(x_{1}, \ldots, x_{q_{1}}, y_{1}, \ldots, y_{q_{2}}\right)= \\
& \quad=\frac{1}{\sqrt{q_{1}}} \sum_{l=1}^{q_{1}} \delta\left(x-x_{l}\right) \phi_{q_{1}-1, q_{2}}\left(x_{1}, \ldots, x_{l-1}, x_{l+1}, \ldots, x_{q_{1}}, y_{1}, \ldots, y_{q_{2}}\right) \\
& \left(\widehat{\psi}_{1}^{-}(x) \phi\right)_{q_{1}, q_{2}}\left(x_{1}, \ldots, x_{q_{1}}, y_{1}, \ldots, y_{q_{2}}\right) \\
& \quad=\sqrt{q_{1}+1} \phi_{q_{1}+1, q_{2}}\left(x, x_{1}, \ldots, x_{q_{1}}, y_{1}, \ldots, y_{q_{2}}\right) \\
& \left(\widehat{\psi}_{2}^{+}(y) \phi\right)_{q_{1}, q_{2}}\left(x_{1}, \ldots, x_{q_{1}}, y_{1}, \ldots, y_{q_{2}}\right) \\
& \quad=\frac{1}{\sqrt{q_{2}}} \sum_{l=1}^{q_{1}} \delta\left(y-y_{l}\right) \phi_{q_{1}, q_{2}-1}\left(x_{1}, \ldots, x_{q_{1}}, y_{1}, \ldots, y_{l-1}, y_{l+1}, \ldots, y_{q_{2}}\right)
\end{aligned}
$$




$$
\begin{aligned}
& \left(\widehat{\psi}_{2}^{-}(y) \phi\right)_{q_{1}, q_{2}}\left(x_{1}, \ldots, x_{q_{1}}, y_{1}, \ldots, y_{q_{2}}\right) \\
& \quad=\sqrt{q_{2}+1} \phi_{q_{1}, q_{2}+1}\left(x_{1}, \ldots, x_{q_{1}}, y, y_{1}, \ldots, y_{q_{2}}\right) .
\end{aligned}
$$

Оператором заряда $\widehat{Q}$ назьвается оператор в $\mathscr{H}$ вида

$$
\widehat{Q}=\int d x \widehat{\psi}_{1}^{+}(x) \widehat{\psi}_{1}^{-}(x)-\int d x \widehat{\psi}_{2}^{+}(x) \widehat{\psi}_{2}^{-}(x) .
$$

Пусть $\widehat{A}$ - оператор в пространстве $\mathscr{H}$, коммутируюший с оператором заряда $\widehat{Q}$. Надвторично квантованным оператором $\overline{\widehat{A}}$ будем называть следующий оператор в $\mathscr{F}$

$$
\widehat{\widehat{A}}=A\left[\widehat{b}_{1}^{+}(x, j), \widehat{b}_{1}^{-}(x, j), \widehat{b}_{2}^{+}(y, i), \widehat{b}_{2}^{-}(y, i), \widehat{B}^{+}(z, w), \widehat{B}^{-}(z, w)\right] .
$$

Функционал $A\left[b_{1}^{*}(\cdot), b_{1}(\cdot), b_{2}^{*}(\cdot), b_{2}(\cdot), B^{*}(\cdot), B(\cdot)\right]$ имеет вид

(4) $\quad A\left[b_{1}^{*}(\cdot), b_{1}(\cdot), b_{2}^{*}(\cdot), b_{2}(\cdot), B^{*}(\cdot), B(\cdot)\right]=\operatorname{Sp}\left(\widehat{A} \widehat{\rho}\left[b_{1}^{*}(\cdot), b_{1}(\cdot), b_{2}^{*}(\cdot), b_{2}(\cdot), B^{*}(\cdot), B(\cdot)\right]\right)$

$$
\times \exp \left(-\sum_{j=1}^{\infty} \int d x b_{1}^{*}(x, j) b_{1}(x, j)-\sum_{i=1}^{\infty} \int d y b_{2}^{*}(y, i) b_{2}(y, i)\right),
$$

а $\widehat{\rho}\left[b_{1}^{*}(\cdot), b_{1}(\cdot), b_{2}^{*}(\cdot), b_{2}(\cdot), B^{*}(\cdot), B(\cdot)\right]$ - операторнозначный функционал, который принимает значения в множестве операторов в пространстве $\mathscr{H}$ и имеет вид

$$
\begin{aligned}
\widehat{\rho}\left[b_{1}^{*}(\cdot),\right. & \left.b_{1}(\cdot), b_{2}^{*}(\cdot), b_{2}(\cdot), B^{*}(\cdot), B(\cdot)\right] \\
= & \exp \left\{\left(\frac{1}{2} \iint d z_{1} d w_{1} B\left(z_{1}, w_{1}\right) \widehat{\psi}_{1}^{+}\left(z_{1}\right) \widehat{\psi}_{2}^{+}\left(w_{1}\right)\right)\right. \\
+ & \left(\frac{1}{2} \iint d z_{2} d w_{2} B^{*}\left(z_{2}, w_{2}\right) \widehat{\psi}_{1}^{-}\left(z_{2}\right) \widehat{\psi}^{-}\left(w_{2}\right)\right) \\
+ & \left(\sum_{j=1}^{\infty} \iint d x_{1} d x_{2} b_{1}\left(x_{1}, j\right) b_{1}^{*}\left(x_{2}, j\right) \widehat{\psi}_{1}^{+}\left(x_{1}\right) \widehat{\psi}_{1}^{-}\left(x_{2}\right)\right) \\
+ & \left(\sum_{i=1}^{\infty} \iint d y_{1} d y_{2} b_{2}\left(y_{1}, i\right) b_{2}^{*}\left(y_{2}, i\right) \widehat{\psi}_{2}^{+}\left(y_{1}\right) \widehat{\psi}_{2}^{-}\left(y_{2}\right)\right) \\
& \left.-\int d x \widehat{\psi}_{1}^{+}(x) \widehat{\psi}_{1}^{-}(x)-\int d y \widehat{\psi}_{2}^{+}(y) \widehat{\psi}_{2}^{-}(y)\right\} .
\end{aligned}
$$

Вторично квантованному оператору $\widehat{A}$ естественным образом ставится в соответствие оператор $\widehat{\widehat{A}}$ в пространстве $\mathscr{F}^{B}$ такой, что

(6) $(\widetilde{\widehat{A}} \Phi)_{q_{1}, q_{2}, m}\left(x_{1}, j_{1}, \ldots, x_{q_{1}}, j_{q_{1}}, y_{1}, i_{1}, \ldots, y_{q_{2}}, i_{q_{2}}, x_{q_{1}+1}, y_{q_{2}+1}, \ldots, x_{q_{1}+m}, y_{q_{2}+m}\right)$

$$
=\left(\widehat{A} \phi\left(j_{1}, \ldots, j_{q_{1}}, i_{1}, \ldots, i_{q_{2}}\right)\right)_{q_{1}+m, q_{2}+m}\left(x_{1}, \ldots, x_{q_{1}+m}, y_{1}, \ldots, y_{q_{2}+m}\right) .
$$

Имеет место следующая теорема

ТЕорема. Проекция надвторично квантованного оператора $\overline{\widehat{A}}$ на бозонное подпространство равна $\widetilde{\widehat{A}}$.

Аналогично тому, как это было сделано в работах [1], [2] для систем с сохраняющимся числом частищ, введем оператор надвторично квантованной энтропии $\overline{\widehat{S}}$ для систем с сохраняющимся зарядом.

\section{СПИСОК ЛИТЕРАТУРЫ}

[1] Маслов В. П. // ТМФ. 2000. Т. 125. №2. С. 297-314. [2] Маслов В. П. // Функц. анализ и его прил. 2000. Т. 34. № 4. С. 35-48. [3] Березин Ф. А. Метод вторичного квантования. М.: Наука, 1986. 